\title{
IR Spectroscopy and Imaging of IRAS 16342-3814
}

\author{
C. Dijkstra, L. B. F. M. Waters, F. Kemper, A. de Koter, \\ S. Hony, C. Dominik
}

Astronomical Institute 'Anton Pannekoek', University of Amsterdam, Kruislaan 403, 1098 SJ Amsterdam, The Netherlands

\author{
A. Zijlstra, M. Matsuura \\ Dept. of Physics, UMIST, Sackville Street, P.O. Box 88, Manchester \\ $M 601 Q D, U K$
}

\section{Introduction.}

The late stages of stellar evolution of stars with low or intermediate mass $\left(1 \mathrm{M}_{\odot}\right.$ to $8 \mathrm{M}_{\odot}$ ) are characterized by extensive mass loss, which can be traced in their circumstellar environment. Here we consider the case of IRAS 16342-3814, a bipolar proto planetary nebula which shows very high velocity $\left(\sim 50 \mathrm{~km} \mathrm{~s}^{-1}\right)$ $\mathrm{OH}$ maser emission (Sahai et al. 1999). We present the complete Infrared Space Observatory (ISO) spectrum and an infrared TIMMI2 Q-band image of this source. Based on these data we discuss the composition and geometry of the circumstellar dust in IRAS 16342-3814.

\section{Overview of the observations and preliminary discussion.}

The ISO spectrum of IRAS 16342-3814 (see figure 1, left panel) is characteristic of that of an extremely reddened OH/IR type Asymptotic Giant Branch (AGB) star, perhaps the reddest observed until now. Saturated amorphous silicate absorption features are seen at 10 and $20 \mu \mathrm{m}$, together with crystalline silicate absorption features up to $45 \mu \mathrm{m}$. No other $\mathrm{OH} / \mathrm{IR}$ star is known to have crystalline silicate features in absorption at these wavelengths. A $45 \mu \mathrm{m}$ feature of crystalline water ice is also found in absorption.

The behaviour of the amorphous and crystalline silicate and crystalline water ice features suggests that IRAS 16342-3814 must have an extremely large mass-loss rate. Indeed, a preliminary radiative transfer calculation of the circumstellar envelope suggests that the mass loss rate may be as large as $10^{-3}$ $\mathrm{M}_{\odot} \mathrm{yr}^{-1}$. Such mass loss rates are only expected during the final stages of the AGB phase (the super wind phase) after which the star turns into a protoplanetary nebula or post-AGB star.

Optical images, taken at 0.55 and $0.80 \mu \mathrm{m}$ with the Hubble Space Telescope (HST), reveal a bipolar reflection nebula, with a dark equatorial waist separating the two lobes of the nebula and obscuring the central star. These images suggest that IRAS 16342-3814 is a protoplanetary nebula (Sahai et al. 1999). Combined with our observation of IRAS 16342-3814 still having an extremely reddened $\mathrm{OH} / \mathrm{IR}$ type spectrum, we therefore suggest that the star must be a very young 

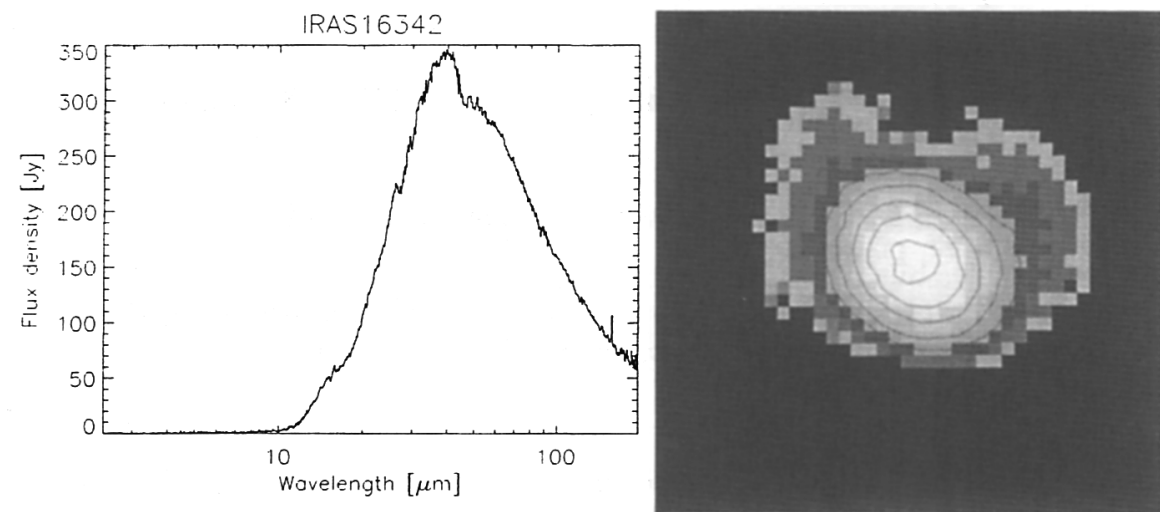

Figure 1. The ISO spectrum (left panel) and the deconvolved TIMMI2 Q-band image (right panel) of IRAS 16342-3814. The total field of view of the TIMMI2 image measures $8 " \times 8$ " and is centered on the source. North is down, east is right. The spectrum of IRAS 16342-3814 is that of an extremely reddened OH/IR star. The source is elongated in the Q-band (see contours) and shows extended emission.

post-AGB star, perhaps the youngest one observed at present. This makes IRAS 16342-3814 a very interesting object for the study of the transition between (OH/IR-type) AGB stars and post-AGB stars.

We observed IRAS 16342-3814 using TIMMI2 at the ESO 3.6m telescope at La Silla (Chile). In figure 1 (right panel) we present the Q-band $(20 \mu \mathrm{m})$ image. The image reveals an elongated structure approximately aligned with the bipolar nebula seen in the HST images and is about 3" along its major axis. The relatively close alignment suggests a high optical depth of dust towards the central star, again indicating a high mass-loss rate. The image also shows a halo around IRAS 16342-3814, most likely a remnant from a period of enhanced, but more spherically symmetric, mass loss during the previous AGB phase.

\section{References}

Sahai, R., Hekkert, P., Morris, M., Zijlstra, A., Likkel, L. 1999, ApJ, 514, L115 